\title{
latrogenically Displaced Renal Calculus During Percutaneous Lithotripsy That Mimicked Duodenal Perforation by Resembling Leaked Oral Contrast
}

\author{
Bozkurt Gulek ${ }^{\mathrm{a}, \mathrm{d}}$, Omer Ozcaglayan ${ }^{\mathrm{a}}$, Cenk Murat Yazici ${ }^{\mathrm{b}}$, \\ Tugba Ilkem Kurtoglu Ozcaglayan ${ }^{\mathrm{c}}$
}

\begin{abstract}
Percutaneous lithotripsy (nephrolithotripsy) is an interventional procedure in which renal calculi are removed from the kidney through a tube which is inserted percutaneously. The procedure removes the necessity of surgery, but still bears the risk of certain complications, including displacement of calculi or their fragments. We present a 56-year-old female patient who was brought to the radiology department with the suspicion of duodenal perforation. The patient had undergone a percutaneous intervention for lithotripsy, but things had gone rather unlucky and the stone had been mobilized out from its original location at the lower pole of the right kidney, by the impact of a stiff nelaton catheter tip used during the intervention. Following the intervention, she had developed an upper abdominal pain. At computed tomography (CT) obtained with oral contrast with the suspicion of a duodenal perforation, a fluid collection and a hyper-dense focus were noted at the near vicinity of the duodenum. The density was originally thought to represent extravasated oral contrast from the duodenum, but later it was found to be due to the calculus that was driven out from the right kidney during the interventional attempt to perform a percutaneous lithotripsy. Renal calculus displacement may be a serious complication of lithotripsy, leading to certain misdiagnoses including hollow-organ penetration. Care and commanding know-how of the procedure are of utmost importance in dealing with such situations.
\end{abstract}

Keywords: Lithotripsy; Complications; Renal calculus

\footnotetext{
Manuscript accepted for publication May 19, 2014

${ }^{a}$ Namik Kemal University School of Medicine, Department of Radiology, Tekirdag, Turkey

${ }^{b}$ Namik Kemal University School of Medicine, Department of Urology, Tekirdag, Turkey

${ }^{\mathrm{c}}$ Tekirdag State Hospital, Department of Radiology, Tekirdag, Turkey

${ }^{\mathrm{d}}$ Correponding Author: Bozkurt Gulek, Namik Kemal University School of Medicine, Department of Radiology, Tekirdag, Turkey.

Email: bozkurtgulek@yahoo.com
}

doi: http://dx.doi.org/10.14740/jmc1811e

\section{Introduction}

The contemporary methods of therapy for renal calculi include open surgery, percutaneous nephrolithotomy (lithotripsy) and extracorporeal shock wave lithotripsy. Percutaneous lithotripsy is a generally approved means of therapy utilized in the treatment of renal calculi [1]. It is a minimally invasive modality and is preferred to open surgery. Among the advantages of percutaneous lithotripsy are high success rates, short hospital stays and short healing periods [2].

As is true for every kind of intervention, percutaneous lithotripsy, too, has a risk of complications. The most frequent complications encountered in lithotripsy are fever (21-32\%), bleeding necessitating transfusion (11-17\%) and extravasation (7\%). Septicemia (4\%), colonic injury (4\%) and pleural injury (1\%) are seldom encountered major complications. Solid organ and duodenal injuries, too, are among the rare complications [3].

\section{Case Report}

A percutaneous interventional procedure was planned for the removal of a small calculus sitting at the lower pole of the right kidney. During the intervention, the calculus was thrown out from the kidney by a nelaton catheter used for the intervention. The procedure was aborted at this level of the intervention. Figure 1 is the computed tomography (CT) image obtained right after the lithotripsy attempt, showing the deformed right kidney and a $3 \mathrm{~mm}$ opacity in the near vicinity of the duodenum (arrow), which was mistaken for extravasated oral contrast from the duodenum.

The patient soon developed a pain at the right upper quadrant which also radiated towards her back. Physical examination revealed a defence and rebound at the region. Considering the possibility of a duodenal perforation, a CT examination with oral contrast was performed. CT revealed a site of free fluid together with a $3 \mathrm{~mm}$ opacity at the near vicinity of the second part of the duodenum. Because these density changes were just near the duodenum, a perforation of the duodenum was considered as a strong possibility, and 


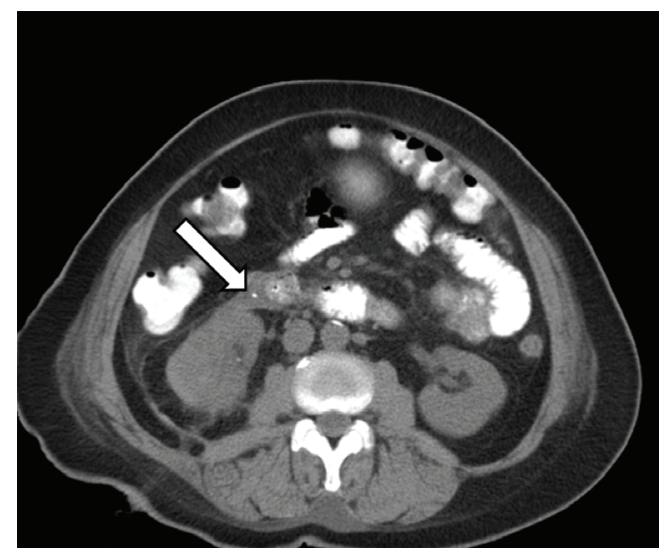

Figure 1. This CT image obtained right after the lithotripsy attempt shows a deformed right kidney together with a $3 \mathrm{~mm}$ opacity in the near vicinity of the duodenum (arrow), which was mistaken for extravasated oral contrast from the duodenum.

the patient was put under strict control and follow-up at the hospital. This CT scan (Fig. 2) demonstrates the tract of the nelaton catheter which was used during the procedure (black arrow). The white arrow points to the displaced calculus which was originally thought to represent extravasated oral contrast from the duodenum.

The patient's abdominal pain ceased in a day. On the second day following the initial CT exam, a plain CT scan was performed and the opacity detected near the duodenum did not show any signs of displacement or change in shape, configuration or size. The density was defined as a displaced renal calculus pushed out from the kidney by the nelaton catheter utilized during an attempt of percutaneous lithotripsy. Figure 3 is a CT image which shows the tract of the nelaton catheter through the kidney, and the calculus.

A follow-up plain CT scan obtained after a week demonstrated no change in the size and configuration of the opacity.

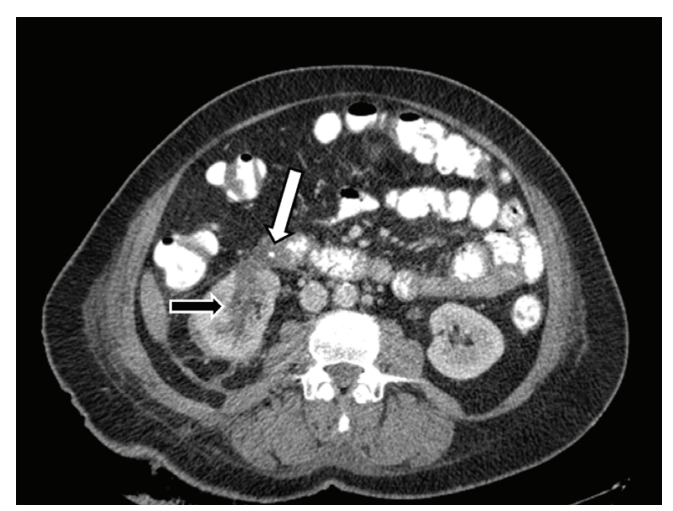

Figure 2. In this CT image, the tract of the nelaton catheter which was used during the procedure may be seen clearly (black arrow). The white arrow points to the displaced calculus which was originally thought to represent extravasated oral contrast from the duodenum.

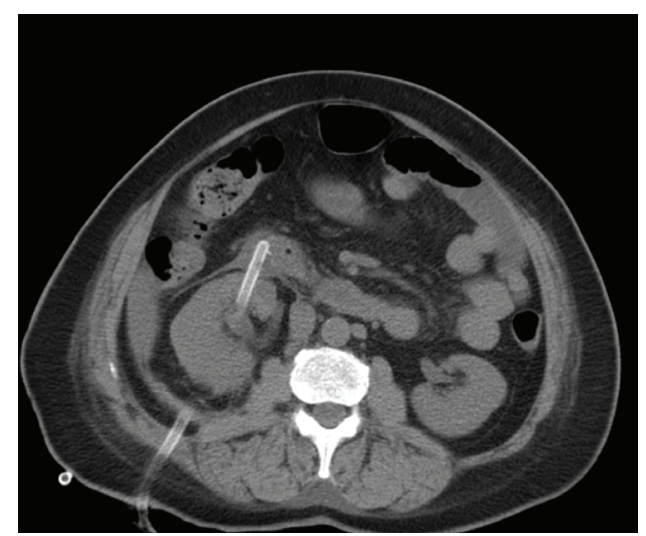

Figure 3. The nelaton catheter, its tract through the kidney and the calculus, can all be seen in a demonstrative fashion in this CT scan.

The patient did well and was discharged from the hospital a few days later.

\section{Discussion}

Organ injury is a rare complication of percutaneous nephrolithotripsy. Pleural injuries constitute $0.3-1.0 \%$ of these complications and are encountered during interventions done for calculi sitting at the upper poles. Usually there is a pneumothorax or a hemothorax in association $[4,5]$. The risk increases in interventions performed above the 12th rib [6].

The colon is the most frequently injured organ in the abdomen, with a complication rate of 0.3-1.0 \% [4]. Patient weakness, over-dilated colonic segment, horseshoe kidney and retro-renal colonic segment, are all among the risk factors [7]. Most of the cases are retro-peritoneal. Peritoneal symptoms are not usually encountered [2].

Intra-peritoneal solid organ injuries are very rare. The most frequently injured organs are the liver and spleen, and these injuries happen more frequently when these organs are enlarged $[1,4]$.

Duodenal injuries are very rare injuries defined in the literature, as a complication of percutaneous interventions of the kidney [8]. Imaging must start with a non-enhanced CT of the abdomen, done without intravenous or oral contrast administration. The reason for this is that extravasated oral contrast and calculus fragments may be mistaken for each other and a differential diagnosis may be impossible. This was exactly what happened with our case. Following the initial non-contrast CT examination, oral contrast must be administered and a CT examination must be done both without and with IV contrast injection. In case of a perforation, orally administered contrast media will extravasate from the duodenum and point to the perforation.

In conclusion, it must be stated that duodenal perforation is a very rare complication of percutaneous nephroli- 
thotomy, and it has a high mortality rate. It is very important to know that the initial CT examination must be done without the use of oral or IV contrast media in order to discriminate displaced fragments of renal calculi from extravasated oral contrast.

\section{Financial Disclosures}

This manuscript did not receive any financial aid. The authors have no disclosures regarding this manuscript.

\section{Author Contributions}

Drs. Bozkurt Gulek and Omer Ozcaglayan planned the study and the structure of the manuscript. Dr. Gulek did the final English writing of the text. Dr. Yazici participated and contributed to the evaluation of the case as the urologist. Dr. Tugba Ozcaglayan aided in the collection of data from the literature.

\section{References}

1. Sofikerim M. Percutaneous nephrolithotomy: in- dications and technique. Erciyes Medical Journal. 2008;30(1):030-036.

2. Telli O, Gulpinar O, Suer E. Complications of percutaneous nephrolithotomy. Turk Urol Sem. 2011;2:336339.

3. Michel MS, Trojan L, Rassweiler JJ. Complications in percutaneous nephrolithotomy. Eur Urol. 2007;51(4):899-906; discussion 906.

4. Taylor E, Miller J, Chi T, Stoller ML. Complications associated with percutaneous nephrolithotomy. Transl Androl Urol. 2012;1(4).

5. Yang WZ, Guo JY, Zhang YQ, Wei RJ, Zhang W. Causes of chest complications and prevention for percutaneous nephrolithotomy lithotripsy. Glob J Medical. 2011;11(1).

6. Lee WJ, Smith AD, Cubelli V, Badlani GH, Lewin B, Vernace F, Cantos E. Complications of percutaneous nephrolithotomy. AJR Am J Roentgenol. 1987;148(1):177180 .

7. Mousavi-Bahar SH, Mehrabi S, Moslemi MK. Percutaneous nephrolithotomy complications in 671 consecutive patients: a single-center experience. Urol J. 2011;8(4):271-276.

8. Lopes Neto AC, Tobias-Machado M, Juliano RV, Lipay MA, Borrelli M, Wroclawski ER. Duodenal damage complicating percutaneous access to kidney. Sao Paulo Med J. 2000;118(4):116-117. 